For reprint orders, please contact: reprints@futuremedicine.com

FOREWORD

Special Focus Issue: Novel Insights into the Pathophysiology and Treatment of Malignant Pleural Mesothelioma

\title{
Malignant pleural mesothelioma: new ideas needed
}

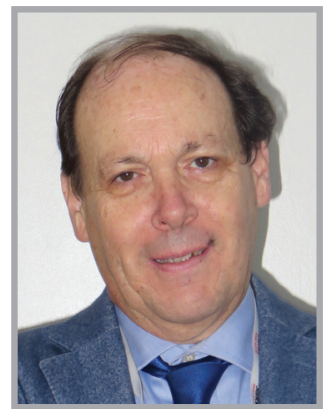

Luciano Mutti* “...this issue of Lung Cancer Management can work

to spur preclinical and clinical scientists to join

their competence and to leave no stones

unturned against this disease."
Malignant pleural mesothelioma (MPM) is a rare and aggressive neoplasm that arises from the mesothelial cells of the serous membrane. In more than $80 \%$ of cases it affects the pleura whereas peritoneal (roughly 20\%), pericardial cavities and the tunica vaginalis are less frequently involved [1,2]. In around $80 \%$ of cases MPM is caused by occupational and environmental asbestos exposure or as a result of second hand exposure. It is more common in men over 65 years of age (sex-ratio 4:1) [1].

Exposure to a Simian Virus 40 (SV40), radiation and erionite have also been shown to play a causative role [3]. The annual incidence of MPM is estimated at around 2000-3000 new cases per year in the USA, $1000-1500$ per year in Great Britain and 1000 per year in Germany [4], while in Europe a quarter of a million deaths of MPM are expected in the next years only among men with occupational exposure. This is due to the long latency period between asbestos exposure and disease onset (from 20 to 40 years) [5]. Moreover, although in most of the countries the use of asbestos is forbidden, MPM incidence is predicted to increase in many developing countries where asbestos is still currently used. Indeed, many experts have announced that we are on the verge of a worldwide epidemic of subjects affected by this cancer.

MPM is characterized by a very poor prognosis with median survival time up to 18 months and the 5-year survival rate is less than 5\% [6]. Survival rate is even shorter for patients with sarcomatous subtype [6].

These daunting figures are mostly due to the fact that MPM is still a difficult cancer to treat and very few drugs, for example, platin combined with antifolates or gemcitabine, show some efficacy against this disease however, unfortunately, their effect on the overall survival is negligible [7].

In earlier stages of nonsarcomatous MPM in patients with good performance status, multimodality therapy is proposed including extrapleural pneumonectomy or extended pleurectomy/decortication, followed by chemotherapy and radiotherapy. Nevertheless there are no randomized clinical trials that can provide a conclusive outcome of this approach as a standard treatment for MPM [7].

Eventually, a standard second-line treatment is still to be defined [7]. Chemotherapy has not shown any significant effect in this setting and even new approaches including those 'biological' have shown disappointing results so far $[8]$.

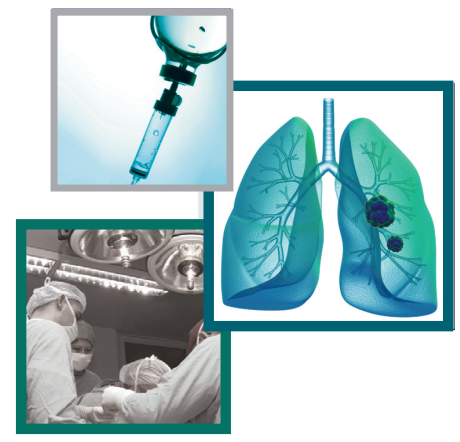

\section{KEYWORDS}

- mesothelioma • standard treatments $\bullet$ translational research
“Malignant pleural mesothelioma
is a rare and aggressive neoplasm
that arises from the mesothelial
cells of the serous membrane.”

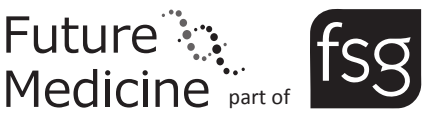




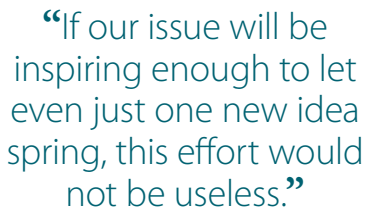

"If our issue will be inspiring enough to let even just one new idea not be useless."
Eleven years ago we published the proceeding of the biannual International Mesothelioma IMIG meeting (IMIG) held in Italy. The title of that article was 'Malignant mesothelioma as both a challenge and an opportunity' and focused on the state of the art of diagnosis and treatment of this cancer concluding that there was still a long and steep way to go [9]. Now after 10 years it is hard to say we have got so far ahead. Unfortunately, recent studies related to molecular biology have not yet proven to be of benefit when translated to the clinical settings [8]. Therefore, more studies, more resources and more ideas are urgently needed to design innovative approaches and to select new potentially active drugs for patients with MPM.

For this very same reason the interest of Lung Cancer Management in a monographic issue on MPM aimed at discussing where we are at and what direction we should take to tackle this worldwide health problem has been warmly welcomed by the scientific community who have been struggling from many years to find a new cure for this cancer.

This issue starts with a clinical input on signs and symptoms of MPM by E Roca and P Astoul addressing the broad and often a specific spectrum of clinical manifestations of this cancer.

The authors also address the physical characteristic of the carcinogenic asbestos fibers.

Oftentimes, pathology and staging of MPM lack of a standardized approach hence the contribute of A Powers and M Carbone offers a critica review of this critical issue.

Particular attention will be paid to both the histologic and immunohistochemical findings observed on tissue biopsy and that should be considered within appropriate clinical and radiologic settings. The crucial dilemma of the true reliability of cytology to diagnose MPM from pleural fluid will also be discussed. Eventually the different system of staging will be critically reviewed.

D Jean and MC Jaurand will be reviewing and discussing the pathophysiology of MPM, a topic clearly entailing discoveries of molecular and genetic mechanisms with potential huge impact in the treatment of this cancer.

MA Archer and R Bueno will contribute with an update on surgery for MPM. The debated issue of patients' selection will be properly discussed to offer the evidences in favor of surgical or nonsurgical approach and on how combining surgery with chemo and radiotherapy.
The same authors will also be discussing how novel biomarkers can support the selection of patients for surgery.

Radiotherapy for MPM has recently drawn the attention [10] even though the role of this treatment for this neoplasm at the moment is rather limited and primarily delivered as adjuvant treatment after either extrapleural pneumonectomy or pleurectomy/decortication and palliative treatment. New delivery systems and new protocols could change this scenario soon.

AS Wolf, KE Rosenzweig and RM Flores will be updating the readers on technical advances that can contribute at the development of safe and effective radiation protocols that can be considered a critical part of treatment in MPM.

Immunotherapy for MPM will be the subject of the contribution by A Khong, A Cook, A Nowak and R Lake.

Some preliminary results provide the evidence that drugs regulating immune check point could be a promising treatment for MPM even though the evidence that this treatment can really improve the survival of these patients is far from being achieved [11].

This contribution will be mostly focused on new laboratory technologies and improvements in clinical procedures such as, for example, adoptive cell transfer therapy along with the research of MPM antigen as potential targets of immunotherapy.

On the whole these articles unveil a scenario with light and shadow even though one cannot perceive that the dark spots unfortunately prevail.

As quoted earlier it is blatant that we do not know enough on this neoplasm, but the reason why its treatment is so disappointing could also lay in something else only very partially known so far.

A proper use of the treatments currently available according to evidence-based medicine is one of the ways to take the gauntlet of this 'untreatable' disease.

As a matter of fact the resistance of MPM to treatments that showed some proof of efficacy in a broad range of tumors is challenging and prompts us to go deeper into the understanding of the mechanism underlying this resistance, and the only option that could change our perspective is to elucidate what is responsible for this difference.

Therefore, this issue of Lung Cancer Management can work to spur preclinical and clinical scientists to join their competence and to leave no stones unturned against this disease. 
If our issue will be inspiring enough to let even just one new idea spring, this effort would not be useless.

\section{Financial \& competing interests disclosure}

The author has no relevant affiliations or financial involvement with any organization or entity with a financial interest in or financial conflict with the subject matter or materials discussed in the manuscript. This includes employment, consultancies, honoraria, stock ownership or options, expert testimony, grants or patents received or pending, or royalties.

No writing assistance was utilized in the production of this manuscript.

\section{References}

1 Ismail-Khan R, Robinson LA, Williams CC Jr, Garrett CR, Bepler G, Simon GR. Malignant pleural mesothelioma: a comprehensive review. Cancer Control 13(4), 255-263 (2006).

2 Segura-González M, Urias-Rocha J, Castelán-Pedraza J. Malignant mesothelioma of the tunica vaginalis: a rare neoplasm - case report and literature review. Clin. Genitourin. Cancer doi:10.1016/j.clgc.2015.05.009 (2015) (Epub ahead of print).

3 Jasani B, Gibbs A. Mesothelioma not associated with asbestos exposure. Arch. Pathol. Lab Med. 136(3), 262-267 (2012).

4 Røe OD, Stella GM. Malignant pleural mesothelioma: history, controversy and future of a manmade epidemic. Eur. Respir. Rev. 24(135), 115-131 (2015).

5 Peto J, Decarli A, La Vecchia C, Levi F, Negri E. The European mesothelioma epidemic. Br. J. Cancer 79(3-4), 666-672 (1999).

6 Fennell DA, Gaudino G, O’Byrne KJ, Mutti $\mathrm{L}$, van Meerbeeck J. Advances in the systemic therapy of malignant pleural mesothelioma. Nat. Clin. Pract. Oncol. 5(3), 136-147 (2008).

7 Scherpereel A, Astoul P, Baas P et al. Guidelines of the European Respiratory Society and the European Society of Thoracic Surgeons for the management of malignant pleural mesothelioma. Eur. Respir. J. 35(3), 479-495 (2010).

8 Pinton G, Manente AG, Tavian D, Moro L, Mutti L. Therapies currently in Phase II trials for malignant pleural mesothelioma. Expert Opin. Investig. Drugs 22(10), 1255-1263 (2013).

9 Mutti L, Broaddus VC. Malignant mesothelioma as both a challenge and an opportunity. Oncogene 23(57), 9155-9161 (2004).

10 Cho BC, Feld R, Leighl N et al. A feasibility study evaluating surgery for mesothelioma after radiation therapy: the "SMART" approach for resectable malignant pleural mesothelioma. J. Thorac. Oncol. 9(3), 397-402 (2014).

11 Guazzelli A, Hussain M, Demonacos MK, Mutti L. Tremelimumab for the treatment of malignant mesothelioma. Expert Opin. Biol. Ther. (2015) (In Press). 